\title{
An alternative response to the off-shell quantum fluctuations: a step forward in resolution of the Casimir puzzle
}

\author{
G. L. Klimchitskaya ${ }^{1,2}$, V. M. Mostepanenko $0^{1,2,3, a}$ \\ ${ }^{1}$ Central Astronomical Observatory at Pulkovo of the Russian Academy of Sciences, Saint Petersburg 196140, Russia \\ ${ }^{2}$ Institute of Physics, Nanotechnology and Telecommunications, Peter the Great Saint Petersburg Polytechnic University, Saint Petersburg 195251, \\ Russia \\ ${ }^{3}$ Kazan Federal University, Kazan 420008, Russia
}

Received: 13 August 2020 / Accepted: 10 September 2020 / Published online: 29 September 2020

(C) The Author(s) 2020

\begin{abstract}
The spatially nonlocal response functions are proposed which nearly coincide with the commonly used local response for electromagnetic fields and fluctuations on the mass shell, but differ significantly for the off-shell fluctuating field. It is shown that the fundamental Lifshitz theory using the suggested response functions comes to an agreement with the measurement data for the Casimir force without neglecting the dissipation of free electrons. We demonstrate that reflectances of the on-shell electromagnetic waves calculated using the nonlocal and commonly employed local responses differ only slightly. The Kramers-Kronig relations for nonlocal response functions possessing the firstand second-order poles at zero frequency are derived, i.e., the proposed response satisfies the principle of causality. An application of these results to resolution of the Casimir puzzle, which lies in the fact that the Lifshitz theory is experimentally consistent only with discarded dissipation, is discussed.
\end{abstract}

\section{Introduction}

Beginning in 2000, much attention is being given to the Casimir force [1] acting between closely spaced uncharged surfaces. This force is caused by the quantum fluctuations (both zero-point and thermal) of the electromagnetic field. It extends familiar van der Waals force [2] to larger separations where the relativistic effects become essential. The general theory of van der Waals and Casimir forces between two material plates developed by Lifshitz $[3,4]$ is in fact semiclassical. It describes electromagnetic fluctuations in the framework of thermal quantum field theory in the Matsubara formulation, but the response of matter to these fluctuations is treated classically by means of the standard continuity bound-

a e-mail: vmostepa@gmail.com (corresponding author) ary conditions where the frequency-dependent dielectric permittivity plays the role of a response function. Taking into consideration that the Casimir effect finds numerous multidisciplinary applications in quantum field theory, physics of elementary particles, gravitation and cosmology, atomic physics, condensed matter physics, as well as in nanotechnology (see, e.g., the monographs [5-11]), it is hardly surprising that the Lifshitz theory was used and cited in thousands of papers.

Over a protracted period of the last 20 years, the Lifshitz theory has been facing a challenge when calculating the Casimir force between metallic surfaces and when comparing the results obtained with the measurement data. According to [12], at room temperature this theory predicts anomalously large thermal effect in the Casimir force even at relatively short separations below $1 \mu \mathrm{m}$ if the electromagnetic response of a metal is described by means of the dissipative Drude dielectric permittivity (or is obtained from the available optical data extrapolated by means of the Drude permittivity down to zero frequency). The measurement data of numerous precise experiments excluded this prediction and were found in good agreement with the Lifshitz theory if the electromagnetic response of a metal is described using the optical data of a metal extrapolated by the dissipationless plasma permittivity (see a review of the first experiments by R.S. Decca in [13]) and later experiments [14-21]). The situation in the field was also reviewed in [22].

Many attempts have been made to explain the about 5\% disagreement between the Lifshitz theory and the measurement data of [13-17] with the role of some unaccounted effects in the surface roughness $[23,24]$, variation of the optical properties of Au films [25], patch potentials [26,27], and by deviations from the proximity force approximation used in computations when one of the plates is replaced with a sphere [28-32]. The facts have been conclusively established 
by the differential force measurement of [18], where the predictions of the Lifshitz theory using the Drude and the plasma responses differ by up to a factor of 1000 . In this experiment, the Lifshitz theory using the Drude response function for calculation of the Casimir force was ultimately excluded over the separation range from 200 to $700 \mathrm{~nm}$, whereas the same theory using the plasma response was found to be in good agreement with the measurement data. Later it was shown that the same is true up to the $1.1 \mu \mathrm{m}$ separation [19-21].

This situation presents a puzzle [33] when it is considered that the Lifshitz theory is based on the first principles of quantum electrodynamics at nonzero temperature and the Drude response function takes proper account of the dissipation of conduction electrons, whereas the plasma response excludes this phenomenon from consideration and is, in fact, applicable only at high frequencies. In the only experiment found in better agreement with the Drude response function at separations of a few micrometers [34], not the Casimir force itself was measured, but up to an order of magnitude larger force of unknown origin. As shown in [35], the interpretation of this experiment is, in fact, uncertain.

It is no less surprising that for metals with perfect crystal lattices the Lifshitz theory violates the third law of thermodynamics (the Nernst heat theorem) when the Drude response is used but is thermodynamically consistent when using the plasma response [36-40]. It has been shown that for lattices with impurities the Casimir entropy calculated using the Drude response satisfies the Nernst theorem [41-43]. However, for a perfect crystal lattice, which is the basic theoretical model of condensed matter physics, the thermodynamic problem remains unresolved.

In the absence of a plausible resolution for this puzzle over a long period, it was hypothesized [21] that the problem might be caused by an incomplete understanding of the response of metal to quantum fluctuations. The point is that the Lifshitz formula for the Casimir pressure (see Sect. 2) depends on the response of a plate material to the quantum fluctuations both on the mass shell and off the mass shell. The spatially local Drude response function is not as fundamental as the Lifshitz theory. It is derived in the framework of kinetic theory and Kubo formula on several assumptions, such as the zero wave vector limit and the relaxation time approximation, which means that the current-current correlation function exponentially decays in time. The Drude function describes reasonably well the response of metal to real electromagnetic field on the mass shell. It is worth mentioning, however, that an experimentalist cannot irradiate a metallic film by the offshell electromagnetic fluctuations and measure the real and imaginary parts of its complex index of refraction. Because of this, there are neither exact theoretical nor direct experimental justifications of an assertion that the response of metals to electromagnetic fluctuations off the mass shell is described by the Drude function.

In this regard, of fundamental interest is graphene governed by the Dirac model $[44,45]$. The polarization tensor of this 2D material was found exactly on the basis of first principles of thermal quantum field theory in the Matsubara formulation [46-51]. The resulting response of graphene to the electromagnetic field is spatially nonlocal, i.e., is described by the two functions depending on both the frequency and the wave vector. The Casimir force in graphene systems, calculated using these functions in the framework of the Lifshitz theory [52], is in good agreement with the measurement data [53], and the Casimir entropy satisfies the Nernst heat theorem $[54,55]$. It seems justified to check the possibility of something similar for metals, although the exact polarization tensor for 3D metallic bodies is, of course, unattainable.

In this paper, we propose the spatially nonlocal phenomenological response functions, which demonstrate nearly the same response as the standard Drude function to the electromagnetic field on the mass shell, but lead to quantitative differences for the off-shell fluctuating field. The response functions suggested here take into account the dissipation of conduction electrons and simultaneously lead to as good agreement with the available measurement data for the Casimir force as does the plasma response. At separations of a few micrometers, the force values, calculated here by using the suggested nonlocal response, are sandwiched between the theoretical predictions obtained using the standard Drude and the plasma responses. We demonstrate that the suggested response satisfies the Kramers-Kronig relations for functions possessing the first- and second-order poles at zero frequency, i.e, it is in agreement with the principle of causality. It also leads to experimentally indistinguishable differences in the reflectances of electromagnetic waves on the mass shell incident on a metallic surface, as compared to the standard Drude response.

The paper is organized as follows: In Sect. 2, we present the summaries of the standard, local, Lifshitz theory and the Lifshitz theory employing the spatially nonlocal response functions. In Sect. 3, the phenomenological nonlocal response functions are introduced which produce an alternative response to the electromagnetic fluctuations off the mass shell. In Sect. 4, we demonstrate that the proposed nonlocal response functions bring the Lifshitz theory in agreement with the measurement data for the Casimir force. Section 5 demonstrates that the suggested nonlocal response leads to nearly the same reflectances of the electromagnetic waves on the mass shell as does the standard Drude function. In Sect. 6, we prove that the proposed nonlocal response is causal and satisfies the Kramers-Kronig relations. In Sect. 7, the reader will find our conclusions and a discussion. 


\section{The Lifshitz theory with spatially local and nonlocal response functions}

According to the Lifshitz theory, the Casimir pressure between two thick metallic plates (semispaces) in thermal equilibrium with the environment at temperature $T$ is given by the Lifshitz formula $[3,4]$

$P(a)=-\frac{k_{B} T}{\pi} \sum_{l=0}^{\infty} \int_{0}^{\infty} q_{l} k_{\perp} d k_{\perp} \sum_{\alpha}\left[\frac{e^{2 a q_{l}}}{r_{\alpha}^{2}\left(\mathrm{i} \xi_{l}, k_{\perp}\right)}-1\right]^{-1}$,

where $k_{B}$ in the Boltzmann constant, $k_{\perp}$ is the magnitude of the projection of wave vector $\boldsymbol{k}$ on the plane of plates, $q_{l}=\left(k_{\perp}^{2}+\xi_{l}^{2} / c^{2}\right)^{1 / 2}, \xi_{l}=2 \pi k_{B} T l / \hbar, l=0,1,2, \ldots$ are the Matsubara frequencies, and the summation in $\alpha$ is over the transverse magnetic (TM) and transverse electric (TE) polarizations of the electromagnetic field (the prime on the summation sign divides the term with $l=0$ by 2 ). The frequently used notation $k_{\perp}$ is due to the fact that the wave vector projection on the plane of plates is perpendicular to the Casimir force which is aligned with the $x_{3}$ axis.

In the standard Lifshitz theory, the reflection coefficients $r_{\alpha}$ have the Fresnel form and are expressed via the frequencydependent dielectric permittivity $\varepsilon_{l}=\varepsilon\left(\mathrm{i} \xi_{l}\right)$ of the plate material at the imaginary Matsubara frequencies

$r_{\mathrm{TM}}\left(\mathrm{i} \xi_{l}, k_{\perp}\right)=\frac{\varepsilon_{l} q_{l}-k_{l}}{\varepsilon_{l} q_{l}+k_{l}}, \quad r_{\mathrm{TE}}\left(\mathrm{i} \xi_{l}, k_{\perp}\right)=\frac{q_{l}-k_{l}}{q_{l}+k_{l}}$,

where $k_{l}=\left(k_{\perp}^{2}+\varepsilon_{l} \xi_{l}^{2} / c^{2}\right)^{1 / 2}$. The permittivity $\varepsilon_{l}$ describes the response of a metal to the electromagnetic field. It can be found from the measured imaginary part of the complex index of refraction using the Kramers-Kronig relations [11].

Taking into account that the optical data for the complex index of refraction are available only to some minimum energy (e.g., to $0.1 \mathrm{eV}$ for $\mathrm{Au}$ [56]), at lower energies the data are usually extrapolated by means of the Drude function

$\varepsilon_{D}(\omega)=1-\frac{\omega_{p}^{2}}{\omega(\omega+\mathrm{i} \gamma)}, \quad \varepsilon_{D}\left(\mathrm{i} \xi_{l}\right)=1+\frac{\omega_{p}^{2}}{\xi_{l}\left(\xi_{l}+\gamma\right)}$,

where $\omega_{p}$ is the plasma frequency and $\gamma$ is the relaxation parameter. As discussed in Sect. 1, this approach leads to contradictions with measurements of the Casimir force. The plasma response function is obtained from (3) by putting $\gamma=0$

$\varepsilon_{p}(\omega)=1-\frac{\omega_{p}^{2}}{\omega^{2}}, \quad \varepsilon_{p}\left(\mathrm{i} \xi_{l}\right)=1+\frac{\omega_{p}^{2}}{\xi_{l}^{2}}$.

When it is used for an extrapolation of the optical data, the Lifshitz theory is brought in agreement with experiments on measuring the Casimir force.
The sharp distinction between the response functions (3) and (4) is in the values of the TE reflection coefficient at zero Matsubara frequency. In the case of the Drude response (3), we have from (2)

$r_{\mathrm{TM}, D}\left(0, k_{\perp}\right)=1, \quad r_{\mathrm{TE}, D}\left(0, k_{\perp}\right)=0$,

whereas the plasma response (4) leads to

$$
\begin{aligned}
& r_{\mathrm{TM}, p}\left(0, k_{\perp}\right)=1, \\
& r_{\mathrm{TE}, p}\left(0, k_{\perp}\right)=\frac{k_{\perp} c-\sqrt{k_{\perp}^{2} c^{2}+\omega_{p}^{2}}}{k_{\perp} c+\sqrt{k_{\perp}^{2} c^{2}+\omega_{p}^{2}}} .
\end{aligned}
$$

Just this distinction results in a disagreement between experiment and theory when the Drude response is used for extrapolation of the optical data and in agreement when an extrapolation is made by means of the plasma response. The crucial point in the above is that the Casimir pressure (1) is determined by the electromagnetic fluctuations on the mass shell, for which $k_{\perp} \leq \xi_{l} / c$, and also off the mass shell for which $k_{\perp}>\xi_{l} / c$. In so doing a common response function $\varepsilon_{l}$ is used for both types of fluctuations which are often called the propagating and evanescent waves, respectively. We emphasize that the electromagnetic field in (5) and (6) is characterized by $\xi=0, k_{\perp}>0$ and, thus, is just off the mass shell.

During the last years, the Lifshitz theory was generalized for the bodies made of any material and of arbitrary geometrical shape [57-60]. It was shown that in the case of plane-parallel configurations the Casimir pressure preserves its form (1), but the reflection coefficients may be quite different from the Fresnel ones (2). Specifically, in the presence of spatial dispersion the response of metal to the electromagnetic field is described by a tensor which is determined by the two independent functions - the longitudinal, $\varepsilon^{L}(\omega, \boldsymbol{k})$, and transverse, $\varepsilon^{T}(\omega, \boldsymbol{k})$, dielectric permittivities depending on the frequency $\omega$ and wave vector $\boldsymbol{k}[61,62]$. Here, the longitudinal and transverse electric fields are parallel and perpendicular to $\boldsymbol{k}$, respectively.

With account of spatial dispersion the reflection coefficients on the surfaces of metallic plates in (1) are found by solving the Maxwell equations with appropriate boundary conditions. They are expressed via the surface impedances as [62-65]

$$
\begin{aligned}
& r_{\mathrm{TM}}\left(\mathrm{i} \xi_{l}, k_{\perp}\right)=\frac{c q_{l}-\xi_{l} Z_{\mathrm{TM}}\left(\mathrm{i} \xi_{l}, k_{\perp}\right)}{c q_{l}+\xi_{l} Z_{\mathrm{TM}}\left(\mathrm{i} \xi_{l}, k_{\perp}\right)}, \\
& r_{\mathrm{TE}}\left(\mathrm{i} \xi_{l}, k_{\perp}\right)=\frac{c q_{l} Z_{\mathrm{TE}}\left(\mathrm{i} \xi_{l}, k_{\perp}\right)-\xi_{l}}{c q_{l} Z_{\mathrm{TM}}\left(\mathrm{i} \xi_{l}, k_{\perp}\right)+\xi_{l}} .
\end{aligned}
$$

The surface impedances in turn are connected with the nonlocal dielectric permittivities [63-65] (see also the 
detailed modern rederivation in [66])

$$
\begin{aligned}
& Z_{\mathrm{TM}}\left(\mathrm{i} \xi_{l}, k_{\perp}\right)=\frac{\xi_{l}}{\pi c} \int_{-\infty}^{\infty} \frac{d k_{z}}{k^{2}}\left(\frac{c^{2} k_{\perp}^{2}}{\xi_{l}^{2} \varepsilon_{l}^{L}}+\frac{k_{z}^{2}}{k_{l}^{T^{2}}+k_{z}^{2}}\right), \\
& Z_{\mathrm{TE}}\left(\mathrm{i} \xi_{l}, k_{\perp}\right)=\frac{\xi_{l}}{\pi c} \int_{-\infty}^{\infty} \frac{d k_{z}}{k_{l}^{T^{2}}+k_{z}^{2}},
\end{aligned}
$$

where $k^{2}=k_{\perp}^{2}+k_{z}^{2}$ and

$\varepsilon_{l}^{L, T} \equiv \varepsilon^{L, T}\left(\mathrm{i} \xi_{l}, \boldsymbol{k}\right), \quad k_{l}^{T^{2}} \equiv k_{\perp}^{2}+\varepsilon_{l}^{T} \frac{\xi_{l}^{2}}{c^{2}}$.

If there is no dependence of the dielectric permittivities on $\boldsymbol{k}$, one obtains

$\varepsilon^{L}\left(\mathrm{i} \xi_{l}, 0\right)=\varepsilon^{T}\left(\mathrm{i} \xi_{l}, 0\right)=\varepsilon_{l}, \quad k_{l}^{T}=k_{l}$

and integrals in (8) are easily calculated with the result

$Z_{\mathrm{TM}}\left(\mathrm{i} \xi_{l}, k_{\perp}\right)=\frac{c k_{l}}{\xi_{l} \varepsilon_{l}}, \quad Z_{\mathrm{TE}}\left(\mathrm{i} \xi_{l}, k_{\perp}\right)=\frac{\xi_{l}}{c k_{l}}$.

Then, the substitution of (11) in (7) returns us back to the standard Fresnel reflection coefficients (2).

Below we suggest the phenomenological nonlocal response functions which take dissipation into account and simultaneously bring the Lifshitz theory in agreement with the measurement data.

\section{Phenomenological nonlocal response functions to the on-shell and off-shell fields}

Spatially nonlocal response functions to the electromagnetic field have long been used in the electrodynamics of solids for theoretical description of the optical properties of charge carriers. The nonlocal response functions $\varepsilon^{L}(\omega, \boldsymbol{k})$ and $\varepsilon^{T}(\omega, \boldsymbol{k})$ for a collisionless electron gas have been found in a seminal work by Lindhard [67] within the random phase approximation and generalized with account of collisions in [68]. In the limiting case $\omega \rightarrow 0$ the obtained function $\varepsilon^{L}$ describes the Thomas-Fermi and Debye screening which has deep physical meaning in electrostatics. The spatially nonlocal generalizations of the Drude response function describing the anomalous skin effect have also been found [63] using the Boltzmann equation and used in the Lifshitz theory to calculate the Casimir force $[66,69,70]$. It was shown, however, that at the experimental separations these nonlocal response functions lead to almost the same Casimir forces as the local Drude response and do not bring theory in agreement with the measurement data.

It should be stressed that the spatially nonlocal generalizations of the Drude response mentioned above describe the physical effects occurring in real electromagnetic fields on the mass shell. Keeping in mind that an account of these effects in the Lifshitz theory does not lead to agreement of the theoretical predictions with the measured Casimir forces, below we consider an alternative, phenomenological, response functions which predict nearly the same results as the standard local response for electromagnetic fields on the mass shell but has quite different properties for the off-shell fields.

Broadly speaking, the nonlocal response functions $\varepsilon^{L}$ and $\varepsilon^{T}$ depend on a 3-component vector $\boldsymbol{k}$ (see the examples in $[67,68])$. It should be noted, however, that all the results of this type have been obtained for the case of homogeneous and isotropic media because in the absence of translational invariance it is impossible in the strict sense to define the nonlocal response functions depending on both $\omega$ and $\boldsymbol{k}$. Taking into consideration that in the Casimir effect the translational invariance in the direction perpendicular to parallel plates is violated, an immediate application of the response functions depending on both $\omega$ and $\boldsymbol{k}$ is not warranted [71-74].

To illustrate our conjecture that an agreement between the Lifshitz theory and the measurement data could be restored by modifying the response of a metal to the off-shell fields, we consider the particular case when $\varepsilon^{L}$ and $\varepsilon^{T}$ in (9) depend not on $\boldsymbol{k}$, but on $k_{\perp}$. This would be in line with the fact that in the plane of the Casimir plates the translational invariance is preserved and in direct analogy to the exact response functions of graphene which depend just on $k_{\perp}$ [46-51].

In this particular case, the integrals in (8) are again calculated exactly

$$
\begin{aligned}
Z_{\mathrm{TM}}\left(\mathrm{i} \xi_{l}, k_{\perp}\right) & =\frac{c k_{\perp}}{\xi_{l} \varepsilon_{l}^{L}}+\frac{\xi_{l}}{c\left(k_{l}^{T}+k_{\perp}\right)} \\
& =\frac{c}{\xi_{l}}\left(\frac{k_{\perp}}{\varepsilon_{l}^{L}}+\frac{k_{l}^{T}-k_{\perp}}{\varepsilon_{l}^{T}}\right), \\
Z_{\mathrm{TE}}\left(\mathrm{i} \xi_{l}, k_{\perp}\right) & =\frac{\xi_{l}}{c k_{l}^{T}},
\end{aligned}
$$

and the substitution of these results in (7) leads to the following reflection coefficients

$$
\begin{aligned}
& r_{\mathrm{TM}}\left(\mathrm{i} \xi_{l}, k_{\perp}\right)=\frac{\varepsilon_{l}^{T} q_{l}-k_{l}^{T}-k_{\perp}\left(\varepsilon_{l}^{T}-\varepsilon_{l}^{L}\right)\left(\varepsilon_{l}^{L}\right)^{-1}}{\varepsilon_{l}^{T} q_{l}+k_{l}^{T}+k_{\perp}\left(\varepsilon_{l}^{T}-\varepsilon_{l}^{L}\right)\left(\varepsilon_{l}^{L}\right)^{-1}}, \\
& r_{\mathrm{TE}}\left(\mathrm{i} \xi_{l}, k_{\perp}\right)=\frac{q_{l}-k_{l}^{T}}{q_{l}+k_{l}^{T}} .
\end{aligned}
$$

With account of (10) it is apparent that in the absence of spatial dispersion (13) transforms to the standard Fresnel reflection coefficients (2) commonly used in the Lifshitz theory.

In order to test a feasibility of the approach discussed above, we consider the following alternative response functions which present a nonlocal modification of the Drude 
response (3):

$$
\begin{aligned}
& \tilde{\varepsilon}_{D}^{T}\left(\omega, k_{\perp}\right)=1-\frac{\omega_{p}^{2}}{\omega(\omega+\mathrm{i} \gamma)}\left(1+\mathrm{i} \frac{v^{T} k_{\perp}}{\omega}\right), \\
& \tilde{\varepsilon}_{D}^{L}\left(\omega, k_{\perp}\right)=1-\frac{\omega_{p}^{2}}{\omega(\omega+\mathrm{i} \gamma)}\left(1+\mathrm{i} \frac{v^{L} k_{\perp}}{\omega}\right)^{-1},
\end{aligned}
$$

where $v^{T, L}$ are the constants of the order of Fermi velocity $v_{F}$. For $k_{\perp}=0$, the functions (14) reduce to the standard Drude function $\varepsilon_{D}(\omega)=\tilde{\varepsilon}_{D}^{L}(\omega, 0)=\tilde{\varepsilon}_{D}^{T}(\omega, 0)$.

Note that the term of the order of $v_{F} k_{\perp} / \omega$, added to unity in (14), is the simplest dimensionless quantity which remains negligibly small for the fields on the mass shell. Really, for the on-shell electromagnetic field we have

$\frac{v^{T, L} k_{\perp}}{\omega} \sim \frac{v_{F}}{c} \frac{c k_{\perp}}{\omega} \leq \frac{v_{F}}{c} \ll 1$,

i.e., any variations, as compared to the standard local Drude response, should be only moderate (see Sect. 5). In Sect. 6 we also prove that the response functions $\tilde{\varepsilon}_{D}^{T, L}$ are causal and satisfy the Kramers-Kronig relations (for $\tilde{\varepsilon}_{D}^{T}$ these relations take the form valid for functions having the first- and secondorder poles at $\omega=0$ [11]).

At the pure imaginary Matsubara frequencies the proposed nonlocal response functions (14) take the form

$$
\begin{aligned}
& \tilde{\varepsilon}_{D}^{T}\left(\mathrm{i} \xi_{l}, k_{\perp}\right) \equiv \tilde{\varepsilon}_{D, l}^{T}=1+\frac{\omega_{p}^{2}}{\xi_{l}\left(\xi_{l}+\gamma\right)}\left(1+\frac{v^{T} k_{\perp}}{\xi_{l}}\right), \\
& \tilde{\varepsilon}_{D}^{L}\left(\mathrm{i} \xi_{l}, k_{\perp}\right) \equiv \tilde{\varepsilon}_{D, l}^{L}=1+\frac{\omega_{p}^{2}}{\xi_{l}\left(\xi_{l}+\gamma\right)}\left(1+\frac{v^{L} k_{\perp}}{\xi_{l}}\right)^{-1} .
\end{aligned}
$$

Using (16) and (13), for the values of the reflection coefficients at zero Matsubara frequency (this is the static limit which is off the mass shell) one obtains

$$
\begin{aligned}
& r_{\mathrm{TM}}\left(0, k_{\perp}\right)=\frac{\omega_{p}^{2}}{\omega_{p}^{2}+2 \gamma v^{L} k_{\perp}}, \\
& r_{\mathrm{TE}}\left(0, k_{\perp}\right)=\frac{k_{\perp}-\sqrt{k_{\perp}^{2}+\omega_{p}^{2} v^{T} k_{\perp} \gamma^{-1} c^{-2}}}{k_{\perp}+\sqrt{k_{\perp}^{2}+\omega_{p}^{2} v^{T} k_{\perp} \gamma^{-1} c^{-2}}} .
\end{aligned}
$$

It is seen that for $v^{L}=v^{T}=0$ the coefficients (17) coincide with (5) obtained for the local Drude response. However, for different from zero $v^{L}$ and $v^{T}$ the equations in (17) are in some sense intermediate between (5) and (6) related to the local Drude and plasma responses, respectively. In so doing the contributions of $r_{\mathrm{TM}}\left(0, k_{\perp}\right)$ to the Lifshitz formula (1) defined in (17), on the one hand, and in (5) and (6), on the other hand, are nearly the same, whereas the contributions of $r_{\mathrm{TM}}\left(0, k_{\perp}\right)$ defined in (17) and in (5) are quite different. It might be thought that theoretical predictions of the Lifshitz theory obtained using the suggested nonlocal response functions agree with the measurement data for the Casimir force. In the next section we provide a verification of this assumption.

\section{Comparison between the Lifshitz theory using the alternative response functions and experiments on measuring the Casimir force}

Before comparing experiment with theory, we compare the theoretical Casimir pressures obtained by using the standard Drude response (3), $P_{D}$, and its nonlocal alternative, $\tilde{P}_{D}$. This comparison is made within the separation region from 1 to $7 \mu \mathrm{m}$ between Au plates where the interband transitions do not contribute so that the obtained results are realistic from the experimental point of view. At first, we have calculated the values of the response functions (16) at the pure imaginary Matsubara frequencies at $T=300 \mathrm{~K}$, where for Au $\hbar \omega_{p}=$ $9.0 \mathrm{eV}$ and $\hbar \gamma=35 \mathrm{meV}$ [56]. For the best agreement with the measurement data (see below), the value $v^{T}=7 v_{F}$, where for $\mathrm{Au} v_{F}=1.38 \times 10^{6} \mathrm{~m} / \mathrm{s}$ [75], has been chosen. The computations made by (1) and (13) show that the change in the value of $v^{L}$ in the range from $v^{L}=0$ (the standard Drude response) to $v^{L}=10 v_{F}$ makes only a negligibly small impact on the values of the Casimir pressure.

The computational results are shown in Fig. 1 by the middle lines (a) for $a^{3}\left|\tilde{P}_{D}\right|$ in the separation region from 1 to $3 \mu \mathrm{m}$ and (b) for $\left|\tilde{P}_{D}\right|$ in the region from 3 to $7 \mu \mathrm{m}$ as the functions of separation between the plates. For comparison purposes, the bottom and top lines demonstrate the respective results $P_{D}$ and $P_{p}$ obtained using the standard Drude and plasma response functions, given in (3) and (4). In these cases the reflection coefficients (2) have been used in place of (13).

As is seen in Fig. 1, the Casimir pressures obtained using the alternative nonlocal response are sandwiched between those found using the standard Drude and plasma responses. Physically the differences between $P_{D}$ and $\tilde{P}_{D}$ are caused by the fact that according to (17) for the alternative nonlocal response $r_{\mathrm{TE}}\left(0, k_{\perp}\right) \neq 0$, as is also the case in (6) for the plasma response [we recall that for the local Drude function $r_{\mathrm{TE}}\left(0, k_{\perp}\right)=0$ in accordance with (5)]. In the insets to Fig. 1a, b, the Casimir pressures computed using the alternative nonlocal and the plasma responses are normalized to $P_{D}$ and shown as functions of separation by the bottom and top lines, respectively. From the inset to Fig. 1b, it is seen that at large separations of a few micrometers the alternative nonlocal response predicts a distinctly smaller pressure magnitudes than the plasma response. However, in this separation region direct precise measurements of the Casimir force are not performed yet.

Now we compare theoretical predictions of the Lifshitz theory using the proposed alternative nonlocal response func- 

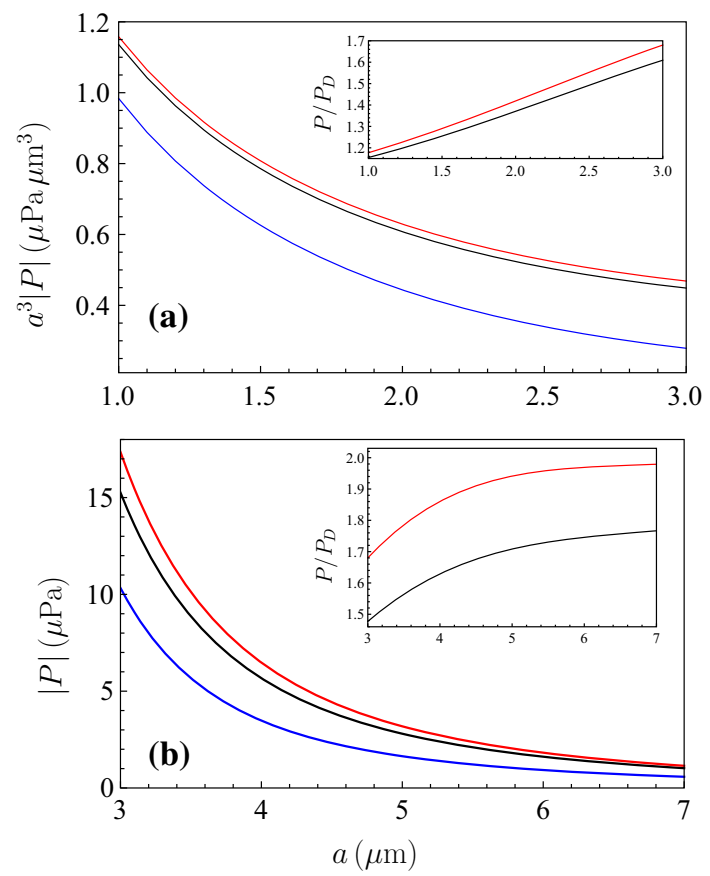

Fig. 1 The magnitudes of the Casimir pressure a multiplied by $a^{3}$ and b on their own, computed using the standard Drude, $P_{D}$, the alternative nonlocal, $\tilde{P}_{D}$, and the plasma, $P_{p}$, responses are shown as functions of separation by the bottom, middle, and top lines, respectively. In the insets, the ratios $\tilde{P}_{D} / P_{D}$ and $P_{p} / P_{D}$ are shown by the bottom and top lines, respectively

tions with the available measurement data. For this comparison, we choose the experiments of [14] and [21] where the gradient of the Casimir force $F_{\text {expt }}^{\prime}$ between a Au-coated sphere of radius $R$ and a Au-coated plate was measured in the separation regions from 235 to $750 \mathrm{~nm}$ and from 0.6 to $2 \mu \mathrm{m}$, respectively (it has been shown [14] that the measurement data of this experiment are in complete agreement with the experimental results of earlier experiments [13] obtained within the same separation region).

The Casimir pressure $P(a)$ between two Au plates given in (1), (13) and (16) in the case of alternative nonlocal response and by (1)-(3) for the standard one was calculated with taken into account interband transitions, which occur at $\hbar \omega>2 \mathrm{eV}$. The contribution of these transitions to the response function influences the Casimir pressure at separations below $1 \mu \mathrm{m}$ and their impact increases with decreasing separation. An inclusion of the interband transitions reduces to a replacement of the unities just after the equality sign on the righthand sides of (3) and (16) with the respective function of the Matsubara frequencies computed by the standard procedure using the optical data for the complex index of refraction of $\mathrm{Au}$ [11]. We have also performed computations with different values of $v^{T}$ and made sure that the value $v^{T}=7 v_{F}$ leads to the best agreement between the experimental results

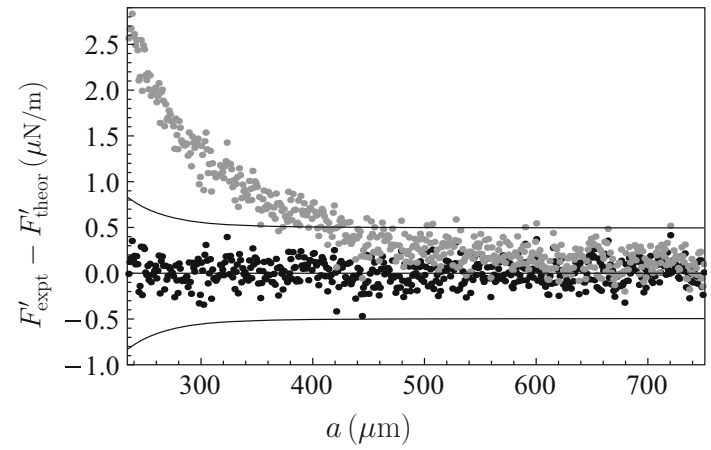

Fig. 2 The differences between experimental [14] and theoretical gradients of the Casimir force computed using the alternative nonlocal (black dots) and the standard Drude (grey dots) responses are shown as functions of separation. The two solid lines indicate borders of the $67 \%$ confidence band

and theoretical predictions (as was noted above, the value of $v^{L}$ makes only a minor impact on the obtained results).

Then, the gradient of the Casimir force between a sphere and a plate was calculated as [21]

$$
\begin{aligned}
F_{\text {theor }}^{\prime}(a)= & -2 \pi R\left[1+\beta(a, R) \frac{a}{R}\right] \\
& \times\left(1+10 \frac{\delta_{s}^{2}+\delta_{p}^{2}}{a^{2}}\right) P(a) .
\end{aligned}
$$

This equation takes into account the rms roughness $\delta_{s}$ and $\delta_{p}$ on the surfaces of a sphere and a plate, respectively. The function $\beta$ takes into account the deviations from the proximity force approximation used in order to adapt the Lifshitz formula (1) derived for two plates to the sphere-plate geometry (see [14,31] for the values of all these quantities).

In Fig. 2 the differences between $F_{\text {expt }}^{\prime}$ measured in [14] and $F_{\text {theor }}^{\prime}$ computed by (18) using the alternative nonlocal and the standard Drude responses are shown by the sets of black and grey dots, respectively. It is seen that the black dots are well inside the $67 \%$ confidence band shown by the two solid lines (the same is true when the plasma response function is used in computations [14], which, however, excludes the dissipation of free electrons from consideration). This means that the alternative nonlocal response is in good agreement with the measurement data. The standard Drude response is experimentally excluded within the separation range from 235 to $420 \mathrm{~nm}$ (see Fig. 2).

In Fig. 3a the differences $F_{\text {expt }}^{\prime}-F_{\text {theor }}^{\prime}$ are shown by using the experimental data of [21] obtained at larger separations. Once again, the Lifshitz theory using the alternative nonlocal response (black dots) is in agreement with the data over the entire separation region from 0.6 to $2 \mu \mathrm{m}$ (the region of $a$ from 1.2 to $2 \mu \mathrm{m}$ is shown on the inset). As is seen in Fig. 3a, the standard Drude response (grey dots) is excluded in the region from 0.6 to $1.1 \mu \mathrm{m}$. For comparison purposes, in Fig. $3 \mathrm{~b}$ we also plot the experimental data as crosses and the 

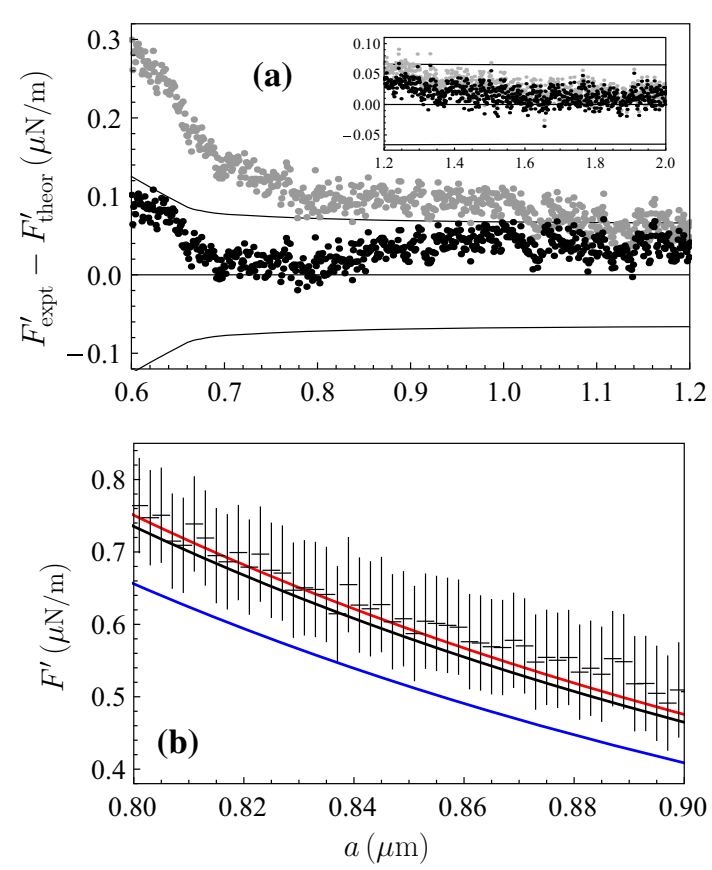

Fig. 3 a The differences between experimental [21] and theoretical gradients of the Casimir force computed using the alternative nonlocal (black dots) and the standard Drude (grey dots) responses are shown as functions of separation. The two solid lines indicate borders of the $67 \%$ confidence band (the region of larger separations is shown in the inset). b The experimental [21] gradients shown as crosses are compared with the theoretical ones computed using the standard Drude, the alternative nonlocal, and the plasma response functions are shown by the bottom, middle, and top lines, respectively

theoretical predictions of the Lifshitz theory using the standard Drude, the alternative nonlocal, and the plasma response functions as the bottom, middle and top lines, respectively. As is seen in Fig. 3b, the theoretical predictions are in agreement with the measurement data when using the alternative nonlocal or the plasma responses, but are excluded by the same data if the standard Drude response is used. Once again, the alternative nonlocal response can be considered as preferential because it takes into account the dissipation of free electrons which is disregarded by the plasma response function.

\section{The alternative nonlocal response and reflection of electromagnetic waves on the mass shell}

According to the above results, the alternative nonlocal response functions introduced in Sect. 3 bring the Lifshitz theory in agreement with measurements of the Casimir force which was unattainable when using the standard (spatially local) Drude response. This has been made possible due to a peculiarity of nonlocal responses to the fluctuating fields off the mass shell. Below we check that the alternative nonlocal functions describe correctly the response of metal to usual electromagnetic waves on the mass shell.
For this purpose, we consider the electromagnetic wave on the mass shell incident under an angle $\theta$ on an Au plate described by the nonlocal response functions (14). Using the relationship $\sin \theta=k_{\perp} c / \omega$, one can present the reflection coefficients (13) calculated along the real frequency axis in the following form:

$$
\begin{aligned}
& r_{\mathrm{TM}}(\omega, \theta)=\frac{\tilde{\varepsilon}_{D}^{T} \cos \theta-\sqrt{\tilde{\varepsilon}_{D}^{T}-\sin ^{2} \theta}+\mathrm{i} \frac{\sin \theta\left(\tilde{\varepsilon}_{D}^{T}-\tilde{\varepsilon}_{D}^{L}\right)}{\tilde{\varepsilon}_{D}^{L}}}{\tilde{\varepsilon}_{D}^{T} \cos \theta+\sqrt{\tilde{\varepsilon}_{D}^{T}-\sin ^{2} \theta}-\mathrm{i} \frac{\sin \theta\left(\tilde{\varepsilon}_{D}^{T}-\tilde{\varepsilon}_{D}^{L}\right)}{\tilde{\varepsilon}_{D}^{L}}}, \\
& r_{\mathrm{TE}}(\omega, \theta)=\frac{\cos \theta-\sqrt{\tilde{\varepsilon}_{D}^{T}-\sin ^{2} \theta}}{\cos \theta+\sqrt{\tilde{\varepsilon}_{D}^{T}-\sin ^{2} \theta}},
\end{aligned}
$$

where now $\tilde{\varepsilon}_{D}^{T, L}=\tilde{\varepsilon}_{D}^{T, L}(\omega, \theta)$. According to (14), one obtains

$$
\begin{aligned}
\tilde{\varepsilon}_{D}^{T}(\omega, \theta)= & 1-\frac{\omega_{p}^{2}}{\omega\left(\omega^{2}+\gamma^{2}\right)}\left[\omega+\gamma \frac{v^{T}}{c} \sin \theta\right. \\
& \left.+\mathrm{i}\left(\gamma-\omega \frac{v^{T}}{c} \sin \theta\right)\right], \\
\tilde{\varepsilon}_{D}^{L}(\omega, \theta)= & 1-\frac{\omega_{p}^{2}}{\omega\left(\omega^{2}+\gamma^{2}\right)}\left[\omega-\gamma \frac{v^{L}}{c} \sin \theta\right. \\
& \left.+\mathrm{i}\left(\gamma+\omega \frac{v^{L}}{c} \sin \theta\right)\right]\left(1+\frac{v^{L^{2}}}{c^{2}} \sin ^{2} \theta\right)^{-1}(20)
\end{aligned}
$$

We have computed the reflectances defined by the alternative nonlocal functions

$$
\begin{aligned}
& \mathcal{R}_{\mathrm{TM}}(\omega, \theta)=\left|r_{\mathrm{TM}}(\omega, \theta)\right|^{2}, \\
& \mathcal{R}_{\mathrm{TE}}(\omega, \theta)=\left|r_{\mathrm{TE}}(\omega, \theta)\right|^{2},
\end{aligned}
$$

where $r_{\mathrm{TM}}$ and $r_{\mathrm{TE}}$ are defined in (19) and (20) with $v^{L}=$ $v^{T}=7 v_{F}$, over the frequency region $\hbar \omega$ from 0.1 to $1 \mathrm{eV}$. In this frequency region, the standard Drude response function is usually used for the interpretation of measured optical data for the complex index of refraction.

We have also computed the standard Drude reflectances

$$
\begin{aligned}
& \mathcal{R}_{\mathrm{TM}, D}(\omega, \theta)=\left|r_{\mathrm{TM}, D}(\omega, \theta)\right|^{2}, \\
& \mathcal{R}_{\mathrm{TE}, D}(\omega, \theta)=\left|r_{\mathrm{TE}, D}(\omega, \theta)\right|^{2},
\end{aligned}
$$

which are also given by (19) and (20), but with $v^{L}=v^{T}=0$.

The relative deviations between the reflectances obtained by using the alternative nonlocal and standard Drude response functions are

$\delta \mathcal{R}_{\mathrm{TM}}(\omega, \theta)=\frac{\mathcal{R}_{\mathrm{TM}}(\omega, \theta)-\mathcal{R}_{\mathrm{TM}, D}(\omega, \theta)}{\mathcal{R}_{\mathrm{TM}, D}(\omega, \theta)}$

for the TM electromagnetic waves and

$$
\delta \mathcal{R}_{\mathrm{TE}}(\omega, \theta)=\frac{\mathcal{R}_{\mathrm{TE}}(\omega, \theta)-\mathcal{R}_{\mathrm{TE}, D}(\omega, \theta)}{\mathcal{R}_{\mathrm{TE}, D}(\omega, \theta)}
$$



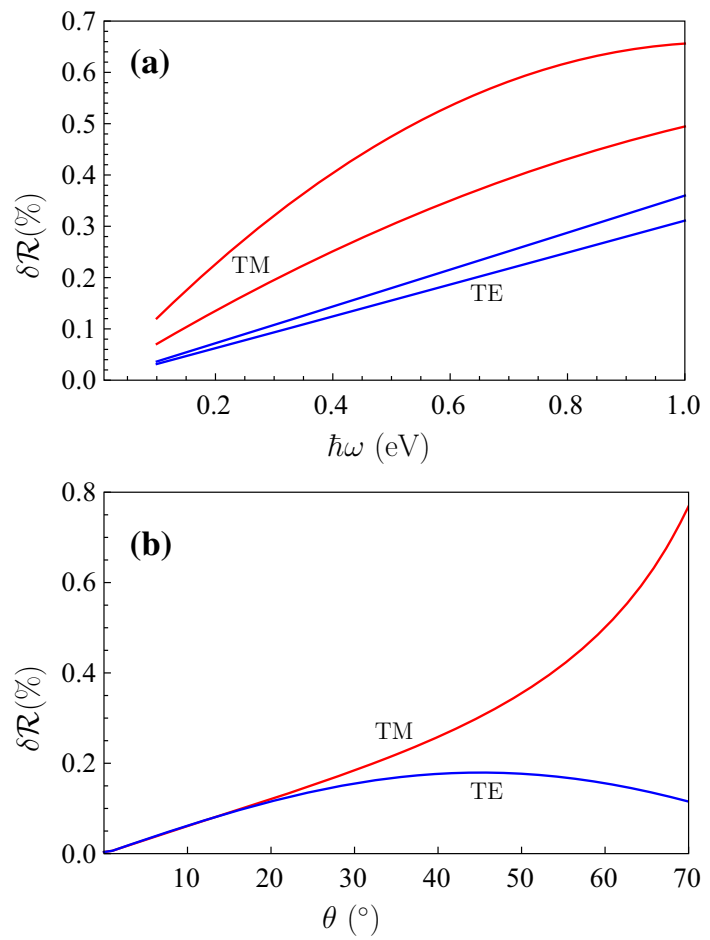

Fig. 4 Relative deviations between the reflectances of electromagnetic waves on the mass shell incident on an Au plate under the angle $\theta$, which are computed using the alternative nonlocal and standard Drude responses, are shown as a the functions of frequency by the top and bottom pairs of lines for the TM and TE polarizations, respectively (the upper and lower lines in the top pair are for $\theta=\pi / 3$ and $\pi / 4$, respectively, and vice versa for the bottom pair) and $\mathbf{b}$ the functions of the incidence angle at $\hbar \omega=0.5 \mathrm{eV}$ by the top and bottom lines for the $\mathrm{TM}$ and TE polarizations, respectively

for the TE ones.

The computational results for $\delta \mathcal{R}_{\mathrm{TM}}$ and $\delta \mathcal{R}_{\mathrm{TE}}$ are presented in Fig. 4. The top and bottom pairs of lines in Fig. 4a show $\delta \mathcal{R}_{\mathrm{TM}}$ and $\delta \mathcal{R}_{\mathrm{TE}}$, respectively, as the functions of $\hbar \omega$. The upper and lower lines in the top pair are for $\theta=\pi / 3$ and $\pi / 4$ angles of incidence, respectively. In the bottom pair of lines, the upper line is for $\theta=\pi / 4$ and the lower line for $\theta=\pi / 3$. The dependences of $\delta \mathcal{R}_{\mathrm{TM}}$ (the top line) and $\delta \mathcal{R}_{\mathrm{TE}}$ (the bottom line) on the incidence angle are illustrated in Fig. $4 \mathrm{~b}$ at $\hbar \omega=0.5 \mathrm{eV}$.

As is seen in Fig. 4, the relative deviations between the reflectances calculated using the alternative nonlocal and the standard Drude response functions do not exceed a fraction of a percent. This is below experimental errors in measuring the optical data for the complex index of refraction and in determination of the parameters $\omega_{p}$ and $\gamma$ in the Drude response. Thus, the suggested alternative nonlocal response leads to the same experimental consequences for the electromagnetic waves on the mass shell as the standard Drude one. In regard to fluctuating fields off the mass shell, the electromagnetic response to them, as discussed in Sect. 1, cannot be immediately measured. Some further circumstantial evidence about it can be obtained only concerning $\varepsilon^{L}$ [62] which, however, does not affect a comparison between the Lifshitz theory and the measurement data (see Sect. 4).

\section{The principle of causality and the Kramers-Kronig relations for the alternative response functions}

As shown in Sects. 4 and 5, the proposed alternative nonlocal response functions not only bring the Lifshitz theory in agreement with measurements of the Casimir force (this has been made possible due to a modified contribution from the off-shell fields), but is also in good agreement with well established physics determined by the on-shell electromagnetic waves. It is pertinent now to make sure that the proposed nonlocal response functions satisfy the fundamental principle of causality formulated mathematically as the KramersKronig relations. According to this principle, the response function in the $(\boldsymbol{x}, t)$ representation must be determined by the field values only at the previous moments $t^{\prime}<t$ and, as a result, its Fourier image must be an analytic function in the upper half plane of complex $\omega$ [61].

We begin with the transverse response function $\tilde{\varepsilon}_{D}^{T}$ defined in the first line of (14). The real and imaginary parts of $\tilde{\varepsilon}_{D}^{T}$ defined along the real frequency axis are given by

$\operatorname{Re} \tilde{\varepsilon}_{D}^{T}\left(\omega, k_{\perp}\right)=1-\frac{\omega_{p}^{2}\left(\omega^{2}+\gamma v^{T} k_{\perp}\right)}{\omega^{2}\left(\omega^{2}+\gamma^{2}\right)}$

and

$\operatorname{Im} \tilde{\varepsilon}_{D}^{T}\left(\omega, k_{\perp}\right)=\frac{\omega_{p}^{2}\left(\gamma-v^{T} k_{\perp}\right)}{\omega\left(\omega^{2}+\gamma^{2}\right)}$.

From (26) it is seen that $\operatorname{Im} \tilde{\varepsilon}_{D}^{T}$ has the pole of the first order, as it holds for the standard Drude function (3), whereas $\operatorname{Re} \tilde{\varepsilon}_{D}^{T}$ in (25) possesses the pole of the second order at zero frequency similar to the plasma response function (4).

It has been known $[61,76]$ that the form of KramersKronig relations depends on whether the dielectric permittivity is regular at $\omega=0$ or it has the poles of the first or second order. The dielectric permittivity $\tilde{\varepsilon}_{D}^{T}$ is the sum of two functions (25) and (26) having the first- and second-order poles at $\omega=0$. Because of this, the standard Kramers-Kronig relations derived for regular at $\omega=0$ functions undergo two respective modifications.

At first, we consider the Kramers-Kronig relation expressing the real part of the dielectric permittivity $\tilde{\varepsilon}_{D}^{T}$ via its imaginary part. The form of this relation is not influenced by the presence of the first-order pole at $\omega=0$ [61]. However, as it was shown previously [76] in a more simple case of the plasma response function (4), due to the presence of the second-order pole in $\operatorname{Re} \tilde{\varepsilon}_{D}^{T}$, one should consider the quantity 
$F\left(\omega, k_{\perp}\right) \equiv 1+\frac{1}{\pi} f_{-\infty}^{\infty} d x \frac{\operatorname{Im} \tilde{\varepsilon}_{D}^{T}\left(x, k_{\perp}\right)}{x-\omega}-\frac{\omega_{p}^{2}}{\omega^{2}} \frac{v^{T} k_{\perp}}{\gamma}$.

The term subtracted on the right-hand side of this equation represents the behavior of $\operatorname{Re} \tilde{\varepsilon}_{D}^{T}$ in the vicinity of $\omega=0$ (the integrals here and below are understood as the principal values).

Substituting (26) in (27), one obtains

$$
\begin{aligned}
F\left(\omega, k_{\perp}\right)= & 1+\frac{\omega_{p}^{2}\left(\gamma-v^{T} k_{\perp}\right)}{\pi} f_{-\infty}^{\infty} \frac{d x}{x\left(x^{2}+\gamma^{2}\right)(x-\omega)} \\
& -\frac{\omega_{p}^{2}}{\omega^{2}} \frac{v^{T} k_{\perp}}{\gamma} .
\end{aligned}
$$

Then, calculating the integrals on the right-hand side of (28), we arrive at

$$
\begin{aligned}
F\left(\omega, k_{\perp}\right)= & 1+\frac{\omega_{p}^{2}\left(\gamma-v^{T} k_{\perp}\right)}{\pi}\left[-\frac{1}{\omega \gamma^{2}} f_{-\infty}^{\infty} \frac{d x}{x}\right. \\
& +\frac{1}{\omega\left(\omega^{2}+\gamma^{2}\right)} f_{-\infty}^{\infty} \frac{d x}{x-\omega} \\
& +\frac{\omega}{\gamma^{2}\left(\omega^{2}+\gamma^{2}\right)} f_{-\infty}^{\infty} \frac{x d x}{x^{2}+\gamma^{2}} \\
& \left.-\frac{1}{\omega^{2}+\gamma^{2}} f_{-\infty}^{\infty} \frac{d x}{x^{2}+\gamma^{2}}\right]-\frac{\omega_{p}^{2}}{\omega^{2}} \frac{v^{T} k_{\perp}}{\gamma} .
\end{aligned}
$$

Taking into account that the first three integrals here are equal to zero and calculating the fourth one, we find

$$
\begin{aligned}
F\left(\omega, k_{\perp}\right) & =1-\frac{\omega_{p}^{2}\left(\gamma-v^{T} k_{\perp}\right)}{\left(\omega^{2}+\gamma^{2}\right) \gamma}-\frac{\omega_{p}^{2}}{\omega^{2}} \frac{v^{T} k_{\perp}}{\gamma} \\
& =1-\frac{\omega_{p}^{2}\left(\omega^{2}+\gamma v^{T} k_{\perp}\right)}{\omega^{2}\left(\omega^{2}+\gamma^{2}\right)}=\operatorname{Re} \tilde{\varepsilon}_{D}^{T}\left(\omega, k_{\perp}\right) .
\end{aligned}
$$

Thus, the first Kramers-Kronig relation for the alternative nonlocal response function $\tilde{\varepsilon}_{D}^{T}$ takes the form

$$
\begin{aligned}
\operatorname{Re} \tilde{\varepsilon}_{D}^{T}\left(\omega, k_{\perp}\right)= & 1+\frac{1}{\pi} f_{-\infty}^{\infty} d x \frac{\operatorname{Im} \tilde{\varepsilon}_{D}^{T}\left(x, k_{\perp}\right)}{x-\omega} \\
& -\frac{\omega_{p}^{2}}{\omega^{2}} \frac{v^{T} k_{\perp}}{\gamma} .
\end{aligned}
$$

Next, we express the imaginary part of $\tilde{\varepsilon}_{D}^{T}$ via its real part. For this purpose we consider the quantity

$$
\begin{aligned}
G\left(\omega, k_{\perp}\right) \equiv & -\frac{1}{\pi} f_{-\infty}^{\infty} \frac{d x}{x-\omega}\left[\operatorname{Re} \tilde{\varepsilon}_{D}^{T}\left(x, k_{\perp}\right)+\frac{\omega_{p}^{2}}{x^{2}} \frac{v^{T} k_{\perp}}{\gamma}\right] \\
& +\frac{\omega_{p}^{2}}{\omega \gamma^{2}}\left(\gamma-v^{T} k_{\perp}\right) .
\end{aligned}
$$

The second term in square brackets of (32) arises because in the vicinity of $\omega=0 \operatorname{Re} \tilde{\varepsilon}_{D}^{T}$ has the second-order pole. For dielectric functions possessing the second-order pole at $\omega=0$, the presence of such a term in the second KramersKronig relation was proven in [76] by the example of the plasma response function (4).

The last term on the right-hand side of (32) represents the behavior of $\operatorname{Im} \tilde{\varepsilon}_{D}^{T}$ in the vicinity of the first-order pole. The term of this kind is present in the second Kramers-Kronig relation for metals [61] and can be interpreted in terms of the transverse conductivity defined as

$\tilde{\varepsilon}_{D}^{T}\left(\omega, k_{\perp}\right)=1+\mathrm{i} \frac{4 \pi \tilde{\sigma}_{D}^{T}\left(\omega, k_{\perp}\right)}{\omega}$.

Using (26), it is easily seen that

$\operatorname{Re} \tilde{\sigma}_{D}^{T}\left(\omega, k_{\perp}\right)=\frac{\omega_{p}^{2}\left(\gamma-v^{T} k_{\perp}\right)}{4 \pi\left(\omega^{2}+\gamma^{2}\right)}$

and, thus, in the static limit,

$\operatorname{Re} \tilde{\sigma}_{D, 0}^{T}\left(k_{\perp}\right)=\lim _{\omega \rightarrow 0} \operatorname{Re} \tilde{\sigma}_{D}^{T}\left(\omega, k_{\perp}\right)=\frac{\omega_{p}^{2}\left(\gamma-v^{T} k_{\perp}\right)}{4 \pi \gamma^{2}}$.

In the local limit $k_{\perp} \rightarrow 0$, (35) transforms into the static conductivity of the standard Drude response function

$\lim _{k_{\perp} \rightarrow 0} \operatorname{Re} \tilde{\sigma}_{D, 0}^{T}\left(k_{\perp}\right) \equiv \sigma_{D, 0}=\frac{\omega_{p}^{2}}{4 \pi \gamma}$.

Substituting (25) in (32), one obtains

$$
\begin{aligned}
G\left(\omega, k_{\perp}\right)= & -\frac{1}{\pi} f_{-\infty}^{\infty} \frac{d x}{x-\omega}\left[1-\frac{\omega_{p}^{2}\left(x^{2}+\gamma v^{T} k_{\perp}\right)}{x^{2}\left(x^{2}+\gamma^{2}\right)}\right. \\
& \left.+\frac{\omega_{p}^{2}}{x^{2}} \frac{v^{T} k_{\perp}}{\gamma}\right]+\frac{\omega_{p}^{2}}{\omega \gamma^{2}}\left(\gamma-v^{T} k_{\perp}\right) .
\end{aligned}
$$

Calculating the integrals on the right-hand side of this equation and again omitting that ones equal to zero, we arrive at

$$
\begin{aligned}
G\left(\omega, k_{\perp}\right) & =-\frac{\omega_{p}^{2} \omega\left(\gamma-v^{T} k_{\perp}\right)}{\gamma^{2}\left(\omega^{2}+\gamma^{2}\right)}+\frac{\omega_{p}^{2}}{\omega \gamma^{2}}\left(\gamma-v^{T} k_{\perp}\right) \\
& =\frac{\omega_{p}^{2}\left(\gamma-v^{T} k_{\perp}\right)}{\omega\left(\omega^{2}+\gamma^{2}\right)}=\operatorname{Im} \tilde{\varepsilon}_{D}^{T}\left(\omega, k_{\perp}\right)
\end{aligned}
$$

in accordance with (26).

Thus, the second Kramers-Kronig relation for the permittivity $\tilde{\varepsilon}_{D}^{T}$ has the form 


$$
\begin{aligned}
\operatorname{Im} \tilde{\varepsilon}_{D}^{T}\left(\omega, k_{\perp}\right)= & -\frac{1}{\pi} \int_{-\infty}^{\infty} \frac{d x}{x-\omega} \\
& \times\left[\operatorname{Re} \tilde{\varepsilon}_{D}^{T}\left(x, k_{\perp}\right)+\frac{\omega_{p}^{2}}{x^{2}} \frac{v^{T} k_{\perp}}{\gamma}\right] \\
& +\frac{4 \pi \operatorname{Re} \tilde{\sigma}_{D, 0}^{T}\left(k_{\perp}\right)}{\omega},
\end{aligned}
$$

where $\operatorname{Re} \tilde{\sigma}_{D, 0}^{T}$ is defined in (35).

In order to derive the Kramers-Kronig relation for the dielectric permittivity $\tilde{\varepsilon}_{D}^{T}$ along the imaginary frequency axis, we consider the quantity

$H\left(\xi, k_{\perp}\right) \equiv 1+\frac{2}{\pi} \int_{0}^{\infty} d x \frac{x \operatorname{Im} \tilde{\varepsilon}_{D}^{T}\left(x, k_{\perp}\right)}{x^{2}+\xi^{2}}+\frac{\omega_{p}^{2}}{\xi^{2}} \frac{v^{T} k_{\perp}}{\gamma}$.

The last term on the right-hand side of this equation should be added because $\operatorname{Re} \tilde{\varepsilon}_{D}^{T}$ possesses the second-order pole at zero frequency [76]. Substituting (26) in (40), one obtains

$$
\begin{aligned}
H\left(\xi, k_{\perp}\right)= & 1+\frac{2 \omega_{p}^{2}\left(\gamma-v^{T} k_{\perp}\right)}{\pi} \int_{0}^{\infty} \frac{d x}{\left(x^{2}+\xi^{2}\right)\left(x^{2}+\gamma^{2}\right)} \\
& +\frac{\omega_{p}^{2}}{\xi^{2}} \frac{v^{T} k_{\perp}}{\gamma} .
\end{aligned}
$$

Now we calculate the integrals on the right-hand side of this equation and obtain

$$
\begin{aligned}
H\left(\xi, k_{\perp}\right) & =1+\frac{\omega_{p}^{2}\left(\gamma-v^{T} k_{\perp}\right)}{(\xi+\gamma) \xi \gamma}+\frac{\omega_{p}^{2}}{\xi^{2}} \frac{v^{T} k_{\perp}}{\gamma} \\
& =1+\frac{\omega_{p}^{2}}{\xi^{2}} \frac{\xi+v^{T} k_{\perp}}{\xi+\gamma}=\tilde{\varepsilon}_{D}^{T}\left(\mathrm{i} \xi, k_{\perp}\right)
\end{aligned}
$$

in accordance to the first line in (16). From (40) and (42) we finally find

$\tilde{\varepsilon}_{D}^{T}\left(\mathrm{i} \xi, k_{\perp}\right)=1+\frac{2}{\pi} \int_{0}^{\infty} d x \frac{x \operatorname{Im} \tilde{\varepsilon}_{D}^{T}\left(x, k_{\perp}\right)}{x^{2}+\xi^{2}}+\frac{\omega_{p}^{2}}{\xi^{2}} \frac{v^{T} k_{\perp}}{\gamma}$.

This equation has the same form as was derived in [76] for the generalized plasma-like response function taking into account the interband transitions in the framework of the oscillator model. As was noted in [61], the presence of the first-order pole makes no impact on the Kramers-Kronig relation expressing the dielectric permittivity along the imaginary frequency axis.

We continue with the longitudinal alternative nonlocal response function defined in the second line of (14). This function can be equivalently written in the form

$\tilde{\varepsilon}_{D}^{L}\left(\omega, k_{\perp}\right)=1-\frac{\omega_{p}^{2}}{(\omega+\mathrm{i} \gamma)\left(\omega+\mathrm{i} v^{L} k_{\perp}\right)}$, i.e. it is an analytic function in the upper half plane of complex frequencies including the entire real frequency axis. Because of this, the permittivity $\tilde{\varepsilon}_{D}^{L}$ satisfies the standard KramersKronig relations derived for insulators [61].

From (44) one finds

$$
\begin{aligned}
& \operatorname{Re} \tilde{\varepsilon}_{D}^{L}\left(\omega, k_{\perp}\right)=1-\frac{\omega_{p}^{2}\left(\omega^{2}-\gamma v^{L} k_{\perp}\right)}{\left(\omega^{2}+\gamma^{2}\right)\left(\omega^{2}+v^{L^{2}} k_{\perp}^{2}\right)}, \\
& \operatorname{Im} \tilde{\varepsilon}_{D}^{L}\left(\omega, k_{\perp}\right)=\frac{\omega \omega_{p}^{2}\left(\gamma+v^{L} k_{\perp}\right)}{\left(\omega^{2}+\gamma^{2}\right)\left(\omega^{2}+v^{L^{2}} k_{\perp}^{2}\right)} .
\end{aligned}
$$

Direct calculation using (45) results in the familiar relations

$\operatorname{Re} \tilde{\varepsilon}_{D}^{L}\left(\omega, k_{\perp}\right)=1+\frac{1}{\pi} f_{-\infty}^{\infty} d x \frac{\operatorname{Im} \tilde{\varepsilon}_{D}^{L}\left(x, k_{\perp}\right)}{x-\omega}$,

$\operatorname{Im} \tilde{\varepsilon}_{D}^{L}\left(\omega, k_{\perp}\right)=-\frac{1}{\pi} f_{-\infty}^{\infty} d x \frac{\operatorname{Re} \tilde{\varepsilon}_{D}^{L}\left(x, k_{\perp}\right)}{x-\omega}$.

The following equality is also valid:

$\tilde{\varepsilon}_{D}^{L}\left(\mathrm{i} \xi, k_{\perp}\right)=1+\frac{2}{\pi} \int_{0}^{\infty} d x \frac{x \operatorname{Im} \tilde{\varepsilon}_{D}^{L}\left(x, k_{\perp}\right)}{x^{2}+\xi^{2}}$.

Thus, being the analytic functions in the upper half plane of complex frequencies, the nonlocal alternatives $\tilde{\varepsilon}_{D}^{T}$ and $\tilde{\varepsilon}_{D}^{L}$ to the Drude response function $\varepsilon_{D}$ considered in this paper are causal and satisfy the Kramers-Kronig relations. The specific form of these relations found above depends on a behavior of the response function in the vicinity of zero frequency.

\section{Conclusions and discussion}

In the foregoing, we have proposed the phenomenological spatially nonlocal response functions to the electromagnetic field which are alternative to the standard Drude function. Unlike nonlocal responses already considered in the literature (see Sect. 1), the suggested ones lead to nearly the same results, as the standard Drude response, in the range of electromagnetic fields and fluctuations on the mass shell, but cause significant differences for the off-shell fields.

We have demonstrated that theoretical predictions of the Lifshitz theory using the optical data of $\mathrm{Au}$ extrapolated down to zero frequency by means of the proposed nonlocal response functions are in a very good agreement with the experimental data on measuring the Casimir force. This can be considered as a step forward in resolution of the Casimir puzzle discussed in Sect. 1. The key advantage of the alternative nonlocal response is that it takes into account the dissipation of conduction electrons, whereas previously it was necessary to simply discard it in order to bring the Lifshitz theory in agreement with the measurement data. It was also 
shown that the suggested response functions lead to nearly the same reflectances of the electromagnetic waves on the mass shell as the standard Drude response. This confirms that the dissipation of free electrons is properly accounted for by the alternative electromagnetic response proposed here.

As mentioned in Sect. 1, the spatially nonlocal alternatives to the Drude function were also found for graphene whose electromagnetic response described by the polarization tensor was derived on the basis of first principles of thermal quantum field theory. As discussed in Sect. 1, the Lifshitz theory using the exact response functions of graphene is in good agreement with measurements of the Casimir force in graphene systems. The same response functions considered on the mass shell describe correctly the reflectances of graphene $[48,49,77]$ and satisfy the Kramers-Kronig relations [78].

The suggested here alternative nonlocal response functions offer a similar situation for Au plates. It is remarkable also that, much like graphene described by the polarization tensor, the Casimir entropy for metallic plates described by these response functions satisfy the Nernst heat theorem. This is eventually connected with the fact that the value of the obtained TE reflection coefficient at zero frequency is not equal to zero [see the second line in (17)], rather than for the standard Drude function where it vanishes (the detailed proof of this statement will be provided elsewhere).

It may be argued that the alternative nonlocal response functions suggested above are somewhat arbitrary and cannot be considered as a rigorous solution of the problem. It is true that these functions are introduced phenomenologically as the simplest example of a situation where the responses to electromagnetic fluctuations on and off the mass shell are dissimilar. Taking into consideration, however, that after twenty years of much work on the subject made by many researchers an agreement between theoretical predictions for the Casimir force with account of dissipation and precise measurements has not been reached, an employment of the phenomenological approach can be considered as warranted.

To conclude, even though the proposed nonlocal response functions do not provide a final resolution of the Casimir puzzle, they may point the new way to its resolution.

Acknowledgements This work was supported by the Peter the Great Saint Petersburg Polytechnic University in the framework of the Program "5-100-2020". V.M.M. was partially funded by the Russian Foundation for Basic Research, grant number 19-02-00453 A. V.M.M. was also partially supported by the Russian Government Program of Competitive Growth of Kazan Federal University.

Data Availability Statement This manuscript has no associated data or the data will not be deposited. [Authors' comment: This is the theoretical manuscript which includes all necessary information and has no new associated experimental data.]
Open Access This article is licensed under a Creative Commons Attribution 4.0 International License, which permits use, sharing, adaptation, distribution and reproduction in any medium or format, as long as you give appropriate credit to the original author(s) and the source, provide a link to the Creative Commons licence, and indicate if changes were made. The images or other third party material in this article are included in the article's Creative Commons licence, unless indicated otherwise in a credit line to the material. If material is not included in the article's Creative Commons licence and your intended use is not permitted by statutory regulation or exceeds the permitted use, you will need to obtain permission directly from the copyright holder. To view a copy of this licence, visit http://creativecomm ons.org/licenses/by/4.0/.

Funded by SCOAP 3 .

\section{References}

1. H.B.G. Casimir, On the attraction between two perfectly conducting plates. Proc. Kon. Ned. Akad. Wet. B 51, 793 (1948)

2. V.A. Parsegian, Van der Waals Forces: A Handbook for Biologists, Chemists, Engineers, and Physicists (Cambridge University Press, Cambridge, 2005)

3. E.M. Lifshitz, The theory of molecular attractive forces between solids. Zh. Eksp. Teor. Fiz. 29, 94 (1955)

4. E.M. Lifshitz, The theory of molecular attractive forces between solids. Sov. Phys. JETP 2, 73 (1956)

5. J. Mahanty, B.W. Ninham, Dispersion Forces (Academic Press, London, 1976)

6. P.W. Milonni, The Quantum Vacuum: An Introduction to Quantum Electrodynamics (Academic Press, San Diego, 1994)

7. M. Krech, The Casimir Effect in Critical Systems (World Scientific, Singapore, 1994)

8. V.M. Mostepanenko, N.N. Trunov, The Casimir Effect and Its Applications (Clarendon Press, Oxford, 1997)

9. K.A. Milton, The Casimir Effect: Physical Manifestations of ZeroPoint Energy (World Scientific, Singapore, 2001)

10. S.Y. Buhmann, Dispersion Forces, I, II (Springer, Berlin, 2012)

11. M. Bordag, G.L. Klimchitskaya, U. Mohideen, V.M. Mostepanenko, Advances in the Casimir Effect (Oxford University Press, Oxford, 2015)

12. M. Boström, Bo E Sernelius, Thermal Effects on the Casimir Force in the 0.1-5 $\mu \mathrm{m}$ Range. Phys. Rev. Lett. 84, 4757 (2000)

13. G.L. Klimchitskaya, U. Mohideen, V.M. Mostepanenko, The Casimir force between real materials: Experiment and theory. Rev. Mod. Phys. 81, 1827 (2009)

14. C.-C. Chang, A.A. Banishev, R. Castillo-Garza, G.L. Klimchitskaya, V.M. Mostepanenko, U. Mohideen, Gradient of the Casimir force between $\mathrm{Au}$ surfaces of a sphere and a plate measured using an atomic force microscope in a frequency-shift technique. Phys. Rev. B 85, 165443 (2012)

15. A.A. Banishev, C.-C. Chang, G.L. Klimchitskaya, V.M. Mostepanenko, U. Mohideen, Measurement of the gradient of the Casimir force between a nonmagnetic gold sphere and a magnetic nickel plate. Phys. Rev. B 85, 195422 (2012)

16. A.A. Banishev, G.L. Klimchitskaya, V.M. Mostepanenko, U. Mohideen, Demonstration of the Casimir force between ferromagnetic surfaces of a Ni-coated sphere and a Ni-coated plate. Phys. Rev. Lett. 110, 137401 (2013)

17. A.A. Banishev, G.L. Klimchitskaya, V.M. Mostepanenko, U. Mohideen, Casimir force between two magnetic metals in comparison with nonmagbetic test bodies. Phys. Rev. B 88, 155410 (2013)

18. G. Bimonte, D. López, R.S. Decca, Isoelectronic determination of the thermal Casimir force. Phys. Rev. B 93, 184434 (2016) 
19. J. Xu, G.L. Klimchitskaya, V.M. Mostepanenko, U. Mohideen, Reducing detrimental electrostatic effects in Casimir-force measurements and Casimir-force-based microdevices. Phys. Rev. A 97, 032501 (2018)

20. M. Liu, J. Xu, G.L. Klimchitskaya, V.M. Mostepanenko, U. Mohideen, Examining the Casimir puzzle with an upgraded AFMbased technique and advanced surface cleaning. Phys. Rev. B 100, 081406(R) (2019)

21. M. Liu, J. Xu, G.L. Klimchitskaya, V.M. Mostepanenko, U. Mohideen, Precision measurements of the gradient of the Casimir force between ultraclean metallic surfaces at larger separations. Phys. Rev. A 100, 052511 (2019)

22. L.M. Woods, D.A.R. Dalvit, A. Tkatchenko, P. Rodriguez-Lopez, A.W. Rodriguez, R. Podgornik, Materials perspective on Casimir and van der Waals interactions. Rev. Mod. Phys. 88, 045003 (2016)

23. P.A. Maia Neto, A. Lambrecht, S. Reynaud, Casimir effect with rough metallic mirrors. Phys. Rev. A 72, 012115 (2005)

24. P.J. van Zwol, G. Palasantzas, J.Th.M. De Hosson, Influence of random roughness on the Casimir force at small separations. Phys. Rev. B 77, 075412 (2008)

25. V.B. Svetovoy, P.J. van Zwol, G. Palasantzas, J.Th.M. De Hosson, Optical properties of gold films and the Casimir force. Phys. Rev. B 77, 035439 (2008)

26. C.C. Speake, C. Trenkel, Forces between conducting surfaces due to spatial variations of surface potential. Phys. Rev. Lett. 90, 160403 (2003)

27. R.O. Behunin, D.A.R. Dalvit, R.S. Decca, C. Genet, I.W. Jung, A. Lambrecht, A. Liscio, D. López, S. Reynaud, G. Schnoering, G. Voisin, Y. Zeng, Kelvin probe force microscopy of metallic surfaces used in Casimir force measurements. Phys. Rev. A 90, $062115(2014)$

28. C.D. Fosco, F.C. Lombardo, F.D. Mazzitelli, Proximity force approximation for the Casimir energy as a derivative expansion. Phys. Rev. D 84, 105031 (2011)

29. G. Bimonte, T. Emig, R.L. Jaffe, M. Kardar, Casimir forces beyond the proximity force approximation. Europhys. Lett. 97, 50001 (2012)

30. G. Bimonte, Going beyond PFA: a precise formula for the sphereplate Casimir force. Europhys. Lett. 118, 20002 (2017)

31. M. Hartmann, G.-L. Ingold, P.A. Maia Neto, Plasma versus Drude modeling of the Casimir force: beyond the proximity force approximation. Phys. Rev. Lett. 119, 043901 (2017)

32. B. Spreng, M. Hartmann, V. Henning, P.A. Maia Neto, G.-L. Ingold, Proximity force approximation and specular reflection: application of the WKB limit of Mie scattering to the Casimir effect. Phys. Rev. A 97, 062504 (2018)

33. G.L. Klimchitskaya, V.M. Mostepanenko, Experiment and theory in the Casimir effect. Contemp. Phys. 47, 131 (2006)

34. A.O. Sushkov, W.J. Kim, D.A.R. Dalvit, S.K. Lamoreaux, Observation of the thermal Casimir force. Nat. Phys. 7, 230 (2011)

35. V.B. Bezerra, G.L. Klimchitskaya, U. Mohideen, V.M. Mostepanenko, C. Romero, Impact of surface imperfections on the Casimir force for lenses of centimeter-size curvature radii. Phys. Rev. B 83, 075417 (2011)

36. V.B. Bezerra, G.L. Klimchitskaya, V.M. Mostepanenko, C. Romero, Violation of the Nernst heat theorem in the theory of thermal Casimir force between Drude metals. Phys. Rev. A 69, 022119 (2004)

37. M. Bordag, I. Pirozhenko, Casimir entropy for a ball in front of a plane. Phys. Rev. D 82, 125016 (2010)

38. M. Bordag, Low Temperature Expansion in the Lifshitz Formula. Adv. Math. Phys. 2014, 981586 (2014)

39. G.L. Klimchitskaya, V.M. Mostepanenko, Low-temperature behavior of the Casimir free energy and entropy of metallic films. Phys. Rev. A 95, 012130 (2017)
40. G.L. Klimchitskaya, C.C. Korikov, Analytic results for the Casimir free energy between ferromagnetic metals. Phys. Rev. A 91, 032119 (2015)

41. S. Boström, B.E. Sernelius, Entropy of the Casimir effect between real metal plates. Physica A 339, 53 (2004)

42. I. Brevik, J.B. Aarseth, J.S. Høye, K.A. Milton, Temperature dependence of the Casimir effect. Phys. Rev. E 71, 056101 (2005)

43. J.S. Høye, I. Brevik, S.A. Ellingsen, J.B. Aarseth, Analytical and numerical verification of the Nernst theorem for metals. Phys. Rev. E 75, 051127 (2007)

44. A.H. Castro Neto, F. Guinea, N.M.R. Peres, K.S. Novoselov, A.K. Geim, The electronic properties of graphene. Rev. Mod. Phys. 81, 109 (2009)

45. M.I. Katsnelson, Graphene: Carbon in Two Dimensions (Cambridge University Press, Cambridge, 2012)

46. M. Bordag, I.V. Fialkovsky, D.M. Gitman, D.V. Vassilevich, Casimir interaction between a perfect conductor and graphene described by the Dirac model. Phys. Rev. B 80, 245406 (2009)

47. I.V. Fialkovsky, V.N. Marachevsky, D.V. Vassilevich, Finitetemperature Casimir effect for graphene. Phys. Rev. B 84, 035446 (2011)

48. M. Bordag, G.L. Klimchitskaya, V.M. Mostepanenko, V.M. Petrov, Quantum field theoretical description for the reflectivity of graphene. Phys. Rev. D 91, 045037 (2015)

49. M. Bordag, G.L. Klimchitskaya, V.M. Mostepanenko, V.M. Petrov, Quantum field theoretical description for the reflectivity of graphene. Phys. Rev. D 93, 089907(E) (2016)

50. M. Bordag, I. Fialkovskiy, D. Vassilevich, Enhanced Casimir effect for doped graphene. Phys. Rev. B 93, 075414 (2016)

51. M. Bordag, I. Fialkovskiy, D. Vassilevich, Enhanced Casimir effect for doped graphene. Phys. Rev. B 95, 119905(E) (2017)

52. G.L. Klimchitskaya, U. Mohideen, V.M. Mostepanenko, Theory of the Casimir interaction for graphene-coated substrates using the polarization tensor and comparison with experiment. Phys. Rev. B 89, 115419 (2014)

53. A.A. Banishev, H. Wen, J. Xu, R.K. Kawakami, G.L. Klimchitskaya, V.M. Mostepanenko, U. Mohideen, Measuring the Casimir force gradient from graphene on $\mathrm{SiO}_{2}$ substrate Phys. Rev. B 87, 205433 (2013)

54. G.L. Klimchitskaya, V.M. Mostepanenko, The Nernst heat theorem for an atom interacting with graphene: Dirac model with nonzero energy gap and chemical potential. Phys. Rev. D 101, $116003(2020)$

55. G.L. Klimchitskaya, V.M. Mostepanenko, Quantum field theoretical description of the Casimir effect between two real graphene sheets and thermodynamics. Phys. Rev. D 102, 016006 (2020)

56. Handbook of Optical Constants of Solids, ed. E.D. Palik (Academic, New York, 1985)

57. T. Emig, N. Graham, R.L. Jaffe, M. Kardar, Casimir forces between arbitrary compact objects. Phys. Rev. Lett. 99, 170403 (2007)

58. O. Kenneth, I. Klich, Casimir forces in a T-operator approach. Phys. Rev. B 78, 014103 (2008)

59. T. Emig, N. Graham, R.L. Jaffe, M. Kardar, Casimir forces between compact objects: The scalar case. Phys. Rev. D 77, 025005 (2008)

60. S.J. Rahi, T. Emig, N. Graham, R.L. Jaffe, M. Kardar, Scattering theory approach to electromagnetic Casimir forces. Phys. Rev. D 80, 085021 (2009)

61. L.D. Landau, E.M. Lifshitz, L.P. Pitaevskii, Electrodynamics of Continuous Media (Pergamon, Oxford, 1984)

62. M. Dressel, G. Grüner, Electrodynamics of Solids: Optical Properties of Electrons in Metals (Cambridge University Press, Cambridge, 2003)

63. K.L. Kliewer, R. Fuchs, Anomalous skin effect for specular electron scattering and optical experiments at non-normal angles of incidence. Phys. Rev. 172, 607 (1968) 
64. V.P. Silin, E.P. Fetisov, Electromagnetic properties of a relativistic plasma, III. Zh. Eksp. Teor. Fiz. 41, 159 (1961)

65. V.P. Silin, E.P. Fetisov, Electromagnetic properties of a relativistic plasma, III. Sov. Phys. JETP 14, 115 (1962)

66. R. Esquivel, V.B. Svetovoy, Correction to the Casimir force due to the anomalous skin effect. Phys. Rev. A 69, 062102 (2004)

67. J. Lindhard, On the properties of a gas of charged particles. Dan. Mat. Fys. Medd. 28, 1 (1954)

68. N.D. Mermin, Lindhard dielectric function in the relaxation time approximation. Phys. Rev. B 1, 2362 (1970)

69. R. Esquivel-Sirvent, V.B. Svetovoy, Nonlocal thin films in calculations of the Casimir force. Phys. Rev. B 72, 045443 (2005)

70. B.E. Sernelius, Effects of spatial dispersion on electromagnetic surface modes associated with a gap between two half spaces. Phys. Rev. B 71, 235114 (2005)

71. Y.S. Barash, V.L. Ginzburg, Electromagnetic fluctuations in a substance and molecular (van der Waals) forces. Usp. Fiz. Nauk 116, 5 (1975)

72. Y.S. Barash, V.L. Ginzburg, Electromagnetic fluctuations in a substance and molecular (van der Waals) forces. Sov. Phys. Uspekhi 18, 305 (1975)
73. V.M. Agranovich, V.L. Ginzburg, Crystal Optics with Spatial Dispersion and Excitons (Springer, Berlin, 1984)

74. G.L. Klimchitskaya, V.M. Mostepanenko, Comment on "Effects of spatial dispersion on electromagnetic surface modes associated with a gap between two half spaces”. Phys. Rev. B 75, 036101 (2007)

75. D. Gall, Electron mean free path in elemental metals. J. Appl. Phys. 119, 085101 (2016)

76. G.L. Klimchitskaya, U. Mohideen, V.M. Mostepanenko, KramersKronig relations for plasma-like permittivities and the Casimir force. J. Phys. A: Math. Theor. 40, 339 (2007)

77. G.L. Klimchitskaya, V.M. Mostepanenko, Reflectivity properties of graphene with a nonzero mass-gap parameter. Phys. Rev. A 93, 052106 (2016)

78. G.L. Klimchitskaya, V.M. Mostepanenko, Kramers-Kronig relations and causality conditions for graphene in the framework of the Dirac model. Phys. Rev. D 97, 085001 (2018) 